\title{
Inhibition of Inflammatory Gene Expression in Keratinocytes Using a Composition Containing Carnitine, Thioctic Acid and Saw Palmetto Extract
}

\author{
Sridar Chittur, ${ }^{1}$ Brian Parr, ${ }^{1}$ and Geno Marcovici ${ }^{2}$ \\ ${ }^{1}$ State University of New York (SUNY), Albany, NY, USA \\ ${ }^{2}$ Advanced Restoration Technologies, Inc., 9035 North 15th Place, Phoenix, AZ 85020, USA \\ Correspondence should be addressed to Geno Marcovici, docgmarcovici@aol.com
}

Received 29 December 2008; Accepted 8 July 2009

Copyright (C) 2011 Sridar Chittur et al. This is an open access article distributed under the Creative Commons Attribution License, which permits unrestricted use, distribution, and reproduction in any medium, provided the original work is properly cited.

\begin{abstract}
Chronic inflammation of the hair follicle (HF) is considered a contributing factor in the pathogenesis of androgenetic alopecia (AGA). Previously, we clinically tested liposterolic extract of Serenoa repens (LSESr) and its glycoside, $\beta$-sitosterol, in subjects with AGA and showed a highly positive response to treatment. In this study, we sought to determine whether blockade of inflammation using a composition containing LSESr as well as two anti-inflammatory agents (carnitine and thioctic acid) could alter the expression of molecular markers of inflammation in a well-established in vitro system. Using a well-validated assay representative of HF keratinocytes, specifically, stimulation of cultured human keratinocyte cells in vitro, we measured changes in gene expression of a spectrum of well-known inflammatory markers. Lipopolysaccharide (LPS) provided an inflammatory stimulus. In particular, we found that the composition effectively suppressed LPS-activated gene expression of chemokines, including CCL17, CXCL6 and $L T B(4)$ associated with pathways involved in inflammation and apoptosis. Our data support the hypothesis that the test compound exhibits anti-inflammatory characteristics in a well-established in vitro assay representing HF keratinocyte gene expression. These findings suggest that 5 -alpha reductase inhibitors combined with blockade of inflammatory processes could represent a novel two-pronged approach in the treatment of AGA with improved efficacy over current modalities.
\end{abstract}

\section{Introduction}

The pathogeneses of benign prostatic hyperplasia $(\mathrm{BPH})$ and androgenetic alopecia (AGA) are mediated in part by the transcriptional pathways downstream of the steroid hormone androgen receptor (AR). The predominant ligand in these tissues is dihydrotestosterone (DHT), which is formed by the conversion of the inactive form of testosterone ( $T$ ) and is catalyzed by the enzyme 5-alpha reductase (5-AR).

Anti-androgens and inhibitors of 5-AR have proven effective in the treatment of BPH as well as AGA, attesting to their common disease mechanisms. Both the pharmaceutical compound, finasteride (Proscar or Propecia) and the liposterolic extract of Serenoa repens (LSESr) have shown efficacy in the treatment of BPH and AGA. Notably, in a direct comparison of LSESr against finasteride, it has been reported that LSESr exhibited a 3-fold greater inhibition of 5-AR in in vitro assays [1].

Finasteride (at a dose of $5 \mathrm{mg}$, as in Proscar) is used as the treatment of choice for $\mathrm{BPH}$, particularly in the USA.
A number of well-controlled studies point to its efficacy in ameliorating the signs and symptoms of $\mathrm{BPH}$ [2]. In large, double-blind, placebo-controlled clinical studies recruiting over 1600 patients, it was shown that the administration of finasteride reduced the size of the prostate by a mean of $22 \%$, following 6 months of therapy [3]. Likewise, multiple wellcontrolled clinical trials reinforce the utility of LSESr in the setting of $\mathrm{BPH}$, predominantly in Europe [4]. Investigators have found that LSESr is well tolerated and has greater efficacy than placebo and similar efficacy to finasteride in improving symptoms in men with BPH [5].

During the course of a clinical trial of Proscar for BPH, it was noted serendipitously that there was a cessation of hair loss in study subjects receiving drug [6]. Therefore, finasteride (at a dose of $1 \mathrm{mg}$; as in Propecia) was subsequently investigated in clinical trials for the treatment of men with AGA. In affected individuals, long-term treatment with finasteride $1 \mathrm{mg}$ /day over 5 years was well-tolerated, led to visible improvements in scalp hair growth and slowed the further progression of hair loss that occurred without 
treatment [7]. LSESr is well known for its role in BPH as a 5AR inhibitor, leading us to postulate a similar effect in AGA.

Previously, we tested LSESr and its glycoside, $\beta$-sitosterol, in subjects with AGA and showed a highly positive response to treatment. The blinded investigative staff assessment reported that $60 \%$ of study subjects dosed with the active study formulation were rated as improved at the conclusion of the trial and established the effectiveness of naturally occurring 5-AR inhibitors against AGA for the first time [8].

Notwithstanding, the common mechanism of androgens in their pathogenesis, several lines of emerging evidence suggest that both $\mathrm{BPH}$ and AGA are also associated with significant dysregulation in the expression of inflammatory cytokines [9]. For example, gene expression profiling of prostate tissue from $\mathrm{BPH}$ patients revealed molecular signatures containing genes associated with inflammation [10]. Likewise, chronic inflammation of the hair follicle (HF) is also considered a contributing factor in AGA [11]. Another recent study reported a relationship between moderate to extensive alopecia and chronic low-grade inflammation [12]. Histologically, it has been shown that in scalp biopsies from AGA patients, sustained follicular inflammation with connective tissue remodeling eventually results in permanent hair loss and, thus, is described as a possible cofactor in the complex etiology of the disorder [11].

On the basis of such findings, the utility of treating $\mathrm{BPH}$-affected patients with anti-inflammatory agents in combination with 5-AR inhibitors is currently under investigation. In one study, combination therapy with alpha(1)adrenergic receptor antagonists [alpha(1)-ARAs] and the 5AR inhibitor finasteride was significantly more effective than either component alone in reducing BPH-related symptoms $(P=.006$ versus doxazosin monotherapy; $P<.001$ versus finasteride monotherapy) and lowering the rate of overall clinical progression $(P<.001$ versus either monotherapy alone) [13]. The greatest efficacy was observed in patients with a markedly enlarged prostate, more severe symptoms and higher PSA levels. These data suggest that the treatment of $\mathrm{BPH}$ is enhanced by the use of anti-inflammatory agents in combination with 5-AR blockade [14]. Taken together, these lines of evidence led us to postulate that inflammation is a rational therapeutic target in pattern hair loss (AGA). In this study, we sought to determine whether blockade of inflammation using a composition containing LSESr, carnitine and thioctic acid (TA) could alter the expression of molecular markers of inflammation in a well-established in vitro system. We found that our compound effectively repressed LPS-activated expression of a number of genes involved in skin inflammation and apoptosis. Our findings suggest that 5-AR inhibitors combined with blockade of inflammatory processes could represent a novel two-pronged approach in the treatment of AGA with improved efficacy over current modalities.

\section{Methods}

2.1. Cell Culture and Challenge. Human keratinocyte cells (HaCaT) were grown in low glucose DMEM supplemented with $10 \%$ FBS, $2 \mathrm{mM}$ L-glutamine, $1 \mathrm{mM}$ sodium pyruvate solution, $0.1 \mathrm{mM}$ NEAA, $100 \mathrm{U} \mathrm{ml}^{-1}$ penicillin, $100 \mu \mathrm{g} \mathrm{ml}^{-1}$ streptomycin at $37^{\circ} \mathrm{C}$ and $5 \% \mathrm{CO}_{2}$. They were subcultured on Day 2 and grown to $80 \%$ confluency on six-well plates.

The HaCaT cells were treated with either $100 \mathrm{ng}$ or $200 \mathrm{ng}$ of LPS (Sigma, St Louis, MO, USA) for $2 \mathrm{~h}$. The media was then replaced with fresh LPS at the same concentration along with the LSESr, TA and carnitine test substance, hereafter designated as TS-050508A $(1: 1000)$. Each condition was carried out in triplicate. The cells were harvested in $1 \mathrm{ml}$ trizol after $22 \mathrm{~h}$. Control wells were treated with $100 \mathrm{ng}$ or $200 \mathrm{ng}$ for $24 \mathrm{~h}$ and harvested in $1 \mathrm{ml}$ trizol. Alternately, the HaCat cells were treated with TS-050508A ( $1: 1000)$ for $2 \mathrm{~h}$. The media was then replaced with either $100 \mathrm{ng}$ or $200 \mathrm{ng}$ of LPS along with fresh TS-050508A ( 1 : 1000). Each condition was carried out in triplicate. The cells were harvested in $1 \mathrm{ml}$ trizol after $22 \mathrm{~h}$. Control wells were treated with TS-050508A $(1: 1000)$ for $24 \mathrm{~h}$ and harvested in $1 \mathrm{ml}$ trizol. Cell viability was determined by trypan blue staining.

2.2. Trypan Blue Staining. The HaCaT cells were treated with $0.5 \mathrm{ml} 0.05 \%$ trypsin-EDTA and placed at $37^{\circ} \mathrm{C}$ in $5 \% \mathrm{CO}_{2}$ for $12 \mathrm{~min}$. The cells were neutralized with $0.5 \mathrm{ml}$ Dulbecco's Modified Eagle's Medium (DMEM) 10\% Fetal Bovine Serum (FBS). $30 \mu \mathrm{l}$ of trypan blue was mixed with $30 \mu \mathrm{l}$ of cell suspension. Approximately $10 \mu \mathrm{l}$ was loaded into a haemocytometer and the cells were counted. Counts were taken at 3, 6, 12 and $24 \mathrm{~h}$ for the No LPS, 100, 200 and $400 \mathrm{ng}$ LPS conditions, respectively. Under our culture conditions and over this concentration range of LPS, the effects of LPS on cell viability, as measured by trypan blue, were variable but there was no consistent decrease in viability as LPS concentration increased. Therefore, we used LPS at the lower end of this concentration range to ensure cell viability. Our findings are consistent with those observed by others [15].

2.3. Nucleic Acids Preparation and PCR-Based Expression Array. Total RNA was isolated from all samples using standard protocols and further cleaned using RNeasy mini columns according to the manufacturer's recommendations (Qiagen, Gaithersburg, MD, USA). The RNA was deemed of good quality for the 260/280, 260/230 and the 28s/18s ribosomal ratios were close to 2 . The RNA (500 ng) was then reverse transcribed to cDNA using the protocols as suggested in the $\mathrm{RT}^{2}$ First strand kit (SA Biosciences, Frederick, MD, USA). The cDNA was then used to prepare a master mix as per the $\mathrm{RT}^{2}$ qPCR kit protocol (SA Biosciences). A $10 \mu \mathrm{l}$ aliquot of this mix was loaded on to the wells in a custom 384-well plate (designed to include most of the genes in the inflammation and cytokine response pathway). The cycle time $\left(C_{t}\right)$ values from these reactions were analyzed using the PCRarray data analysis template to calculate the $\Delta \Delta \mathrm{Ct}$ and the fold change [fold change $=2^{(-\Delta \Delta \mathrm{Ct})}$ ].

2.4. RT-PCR Validation of Results. Equal amount of RNA $(1 \mu \mathrm{g})$ was taken for all samples and reverse transcription was performed using RT $^{2}$ First Strand kit (Cat\# C-03) from SA Biosciences. The total volume of the reaction was $20 \mu \mathrm{l}$, and the final RT product was diluted to a volume of 100 
$\mu$ l. Individual PCR assays were performed with the off-theshelf pre-validated primer pairs from SA Biosciences on the BioRad iCycler iQ using RT ${ }^{2}$ SYBR Green/ROX qPCR Master Mix (Cat\# PA-011, SA Biosciences). PCR assays for each gene target were performed in triplicates for each sample. Each PCR reaction contained cDNA synthesized from $10 \mathrm{ng}$ total RNA. Relative changes in gene expression were calculated using the $\Delta \Delta \mathrm{C}_{\mathrm{t}}$ (threshold cycle) method. Fold change values are calculated using the formula $2^{-\Delta \Delta \mathrm{Ct}}$. There were three biological replicates in each treatment group. Comparisons between groups were made using unpaired Student's $t$-tests with the level of significance set at $P<.05$.

\section{Results}

3.1. Test Compound Suppressed LPS-activated Gene Expression. In this study, we used a well-validated in vitro assay representative of HF keratinocytes, specifically, stimulation of cultured HaCaT cells. We measured changes in gene expression of a spectrum of well-known inflammatory markers, using lipopolysaccharide (LPS) as an inflammatory stimulus. Using untreated cells as a baseline for gene expression, we tested our compound under seven conditions: (i) $100 \mathrm{ng}$ LPS alone (low dose); (ii) $200 \mathrm{ng}$ LPS alone (high dose); (iii) compound alone (1:1000); (iv) compound followed by low-dose LPS; (v) compound followed by high-dose LPS; (vi) low-dose LPS followed by the compound and lastly (vii) high-dose LPS followed by the compound. Overall, we found that treating $\mathrm{HaCaT}$ cell cultures with our compound resulted in reduced LPSmediated inflammatory gene expression without inducing a negative effect on cell viability. In particular, we found that the composition effectively suppressed LPS-activated gene expression of chemokines associated with pathways involved in inflammation and apoptosis.

3.2. Global Expression Analysis Reveals Inhibition of Inflammatory Pathways. The RT ${ }^{2}$ Profiler PCR Array from SuperArray was used to interrogate the effect of the novel composition on inflammation. This array contains 384 wellvalidated genes known to be involved in stromal, endothelial and epithelial inflammatory pathways. The classes of genes represented on the array included the CC ligand chemokines, toll-like receptor genes and their ligands, epithelial cell adhesion molecules, and members of the CXC chemokine family and leukotriene lipid mediators derived from the 5lipoxygenase pathway of arachidonic acid metabolism.

When measured against the untreated cells, we noted statistically significant $(P \leq .05)$ fold changes in the following genes: CCL3, CCL8, CCL17, CCL24, CCR7, CXCL2, CXCL6, IL18RAP, IL1A, IL1B, IL1F10 LTB(4) and TLR4, (Table 1). Below we will focus on three genes, CCL17, CXCL6 and LTB(4) (Figure 1).

3.3. Chemokine Ligand CCL17. In this study, our compound showed modest but statistically significant antiinflammatory activity against CCL17. The HaCaT cells treated with $100 \mathrm{ng}$ LPS displayed a 1.76 up-regulated fold

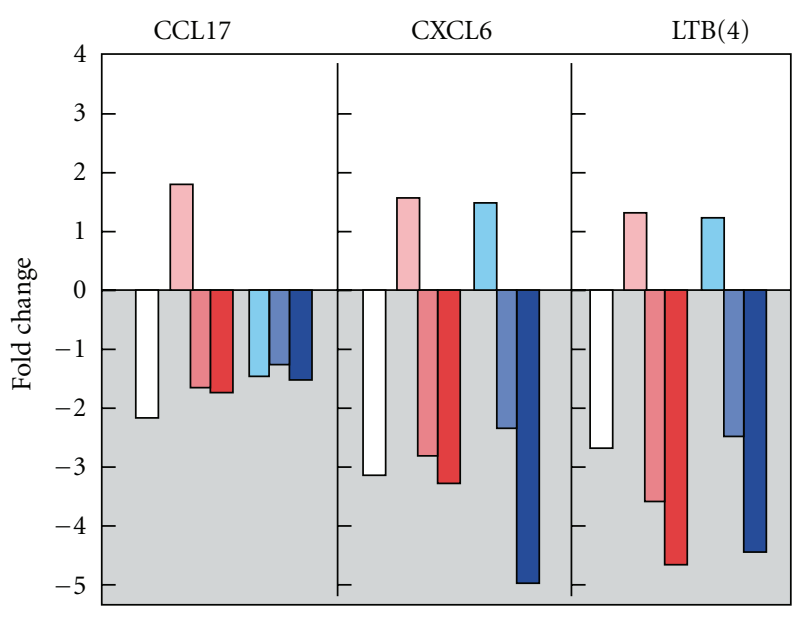

$1: 1000$ drug only

100 ng LPS only

100 ng LPS + drug

drug + 100 ng LPS

200 ng LPS only

200 ng LPS + drug

drug + 200 ng LPS

FIGURE 1: Noteworthy fold change occurring in CCL17, CXCL6 and $\operatorname{LTB}(4)$.

change as compared to untreated controls. Dosing the cells with $200 \mathrm{ng}$ LPS resulted in a down-regulation of CCL17 by a factor of -1.43 -fold. The cells treated with the test compound alone down-regulated expression of CCL17 by a factor of -2.17 from baseline, whereas cells treated with compound followed by low-dose LPS resulted in a downregulation of -1.71 -fold. Overall, the results showed that our compound down-regulated the inflammatory marker CCL17. The test composition also blocked the up-regulation of message, both prior to and subsequent to LPS-mediated challenge.

3.4. CXC Chemokine Ligand, CXCL6. We show statistically significant anti-inflammatory activity in an LPS-mediated model of skin inflammation via the marker CXCL6. The low-dose LPS-only (100 ng) stimulated cells resulted in CXCL6 being up-regulated by 1.57 -fold. When measured against baseline, the test compound alone down-regulated expression of CXCL6 by a factor of -3.09. After incubating the cells with the test compound followed by exposure to low-dose LPS, CXCL6 expression showed a -3.28 -fold change as measured against baseline. These results show a blockade of CXCL6 inflammatory message in the LPSstimulated $\mathrm{HaCaT}$ cells by the test compound.

3.5. Leukotriene B(4). In this study, our test compound showed a noteworthy effect in the leukotriene $[\operatorname{LTB}(4)]$ marker. The HaCaT cells treated with $100 \mathrm{ng}$ LPS inflammatory agonist displayed an up-regulated fold change for 
TABLE 1: Gene expression profile of inflammatory markers expressing statistically significant fold change.

\begin{tabular}{|c|c|c|c|c|c|c|c|c|}
\hline Symbol & Well & $\begin{array}{l}1: 1000 \text { drug } \\
\text { only Fold upor } \\
\text { down- } \\
\text { regulation } \\
1: 1000 \text { drug } \\
\text { only/no } \\
\text { drug no LPS }\end{array}$ & $\begin{array}{l}100 \text { ng LPS only } \\
\text { Fold upor } \\
\text { down- } \\
\text { regulation } \\
100 \text { ng LPS } \\
\text { only/no } \\
\text { drug no LPS }\end{array}$ & $\begin{array}{l}100 \text { ng LPs+ } \\
\text { drug Fold upor } \\
\text { down- } \\
\text { regulation } \\
100 \text { ng } \\
\text { LPS+drug/no } \\
\text { drug no LPS }\end{array}$ & $\begin{array}{l}\text { drug+100 ng } \\
\text { LPS Fold up- or } \\
\text { down- } \\
\text { regulation } \\
\text { drug+100 ng } \\
\text { LPS/no } \\
\text { drug no LPS }\end{array}$ & $\begin{array}{l}200 \text { ng LPS only } \\
\text { Fold upor } \\
\text { down- } \\
\text { regulation } \\
200 \text { ng LPS } \\
\text { only/no } \\
\text { drug no LPS }\end{array}$ & $\begin{array}{l}200 \text { ng LPS+ } \\
\text { drug Fold upor } \\
\text { down- } \\
\text { regulation } \\
200 \text { ng } \\
\text { LPS+drug/no } \\
\text { drug no LPS }\end{array}$ & $\begin{array}{l}\text { drug+200 ng } \\
\text { LPS Fold upor } \\
\text { down- } \\
\text { regulation } \\
\text { drug+200 ng } \\
\text { LPS/no } \\
\text { drug no LPS }\end{array}$ \\
\hline CCL17 & A08 & -2.17 & 1.76 & -1.63 & -1.71 & -1.43 & -1.25 & -1.49 \\
\hline CCL24 & B02 & -2.74 & -4.12 & -1.70 & -1.65 & -3.95 & -5.21 & -3.27 \\
\hline CCL3 & B03 & 2.04 & 1.55 & -1.05 & 2.84 & 1.77 & -1.47 & 1.80 \\
\hline CCL8 & B07 & -1.01 & 2.47 & 1.55 & 1.40 & 2.01 & 1.38 & 1.15 \\
\hline CCR7 & B12 & -2.29 & -1.46 & -1.96 & -1.27 & -3.31 & -1.43 & -1.67 \\
\hline CXCL2 & C08 & -2.18 & -1.35 & -2.22 & -3.34 & -1.41 & -1.27 & -2.34 \\
\hline CXCL6 & $\mathrm{C} 11$ & -3.09 & 1.57 & -2.78 & -3.28 & 1.45 & -2.30 & -4.92 \\
\hline IL18RAP & D10 & -2.37 & -1.01 & -1.13 & 1.55 & 1.41 & 1.20 & 1.31 \\
\hline IL1A & D11 & 2.53 & -1.73 & -1.06 & 1.13 & -1.62 & 2.77 & 2.24 \\
\hline IL1B & D12 & 2.12 & -1.21 & 1.07 & 1.04 & -1.27 & 2.39 & 1.81 \\
\hline IL1F10 & E01 & 3.67 & -2.33 & -5.36 & -4.73 & -3.70 & -3.95 & -5.09 \\
\hline $\operatorname{LTB}(4)$ & F06 & -2.64 & 1.33 & -3.54 & -4.59 & 1.23 & -2.44 & -4.37 \\
\hline TLR4 & G06 & 2.97 & -1.70 & 1.67 & 2.31 & -1.32 & 3.36 & 2.84 \\
\hline
\end{tabular}

Values that have been highlighted showed weak amplification in qPCH; Light gray shadings indicates both control and test samples showed weak amplification; Dark gray shadings indicates only test sample shows weak amplification. Bold text indicates stastically significant fold changes.

LTB(4) of 1.33 when measured against baseline. Gene expression of $\operatorname{LTB}(4)$ showed a down-regulation of -2.64fold in the cells treated with the test compound alone. A $100 \mathrm{ng}$ LPS incubated cells followed by the test compound showed marked change in $\operatorname{LTB}(4)$ expression demonstrating a -4.59 -fold change as measured against baseline. These results show that our compound blocked the LPS-stimulated up-regulation of the inflammatory marker $\operatorname{LTB}(4)$.

In summary, we found that our test compound attenuated expression for markers of inflammation, and specifically for CCL17, CXCL6 and LTB(4). Our data support the hypothesis that the test compound exhibits antiinflammatory characteristics in a well-established in vitro assay representing HF keratinocyte gene expression.

\section{Discussion}

In this study, we sought to demonstrate that a test compound containing LSESr, carnitine and TA represses LPS-activated expression of inflammatory genes using a well-validated in vitro assay representative of $\mathrm{HF}$ keratinocytes, specifically, the LPS-stimulated HaCaT cells $[16,17]$. We assayed for changes in gene expression across a spectrum of well-characterized inflammatory markers and found that our test compound demonstrates anti-inflammatory properties in vitro. Plant extracts or mixtures comprise a repertoire of chemical entities that have pleiotropic effects on cellular physiology. As such, they have great potential in the multi-target approach to diseases $[18,19]$. Microarray analysis of gene expression following exposure of cells to plant extracts can be useful for elucidating the molecular networks impacted by herbal extracts and mixtures [20]. In particular, plantbased therapies have proven efficacious in the treatment of inflammatory skin disorders, such as atopic dermatitis [21].

Here, we found that treating $\mathrm{HaCaT}$ cell cultures with our compound resulted in a statistically significant $(P<$ $.05)$ reduction of LPS-mediated inflammatory gene expression without inducing a negative effect on cell viability. Specifically, we noted that the composition effectively suppressed LPS-activated gene expression of chemokines CCL17, CXCL6 and LTB(4). The over-expression of each of these markers has previously been observed in the setting of inflammation and apoptosis. We focused here on short term and immediate changes in gene expression, using array hybridization, followed by qPCR. The gene expression fold changes we observed were statistically significant. Our approach is supported in the literature by many other examples using the same strategy, in studying acute responses to stimuli such as LPS, in addition to UV irradiation and wounding in the HaCaT cells and keratinocytes [22-25].

Our rationale for selecting CXCL6, CCL17 and LTB(4) for further study relates to their role in inflammatory disorders of the skin, mucosae and epithelia. CXCL6 is a CXC chemokine expressed by macrophages, epithelial and mesenchymal cells during inflammation. Recently, it was shown that the transcriptome of the aging prostate stroma is characterized by the up-regulation of several genes that encode secreted inflammatory mediators, including CXCL6 [26]. Similarly, in the skin, CCL17 (a CC chemokine ligand) is up-regulated under stress, injury or inflammation [27]. 


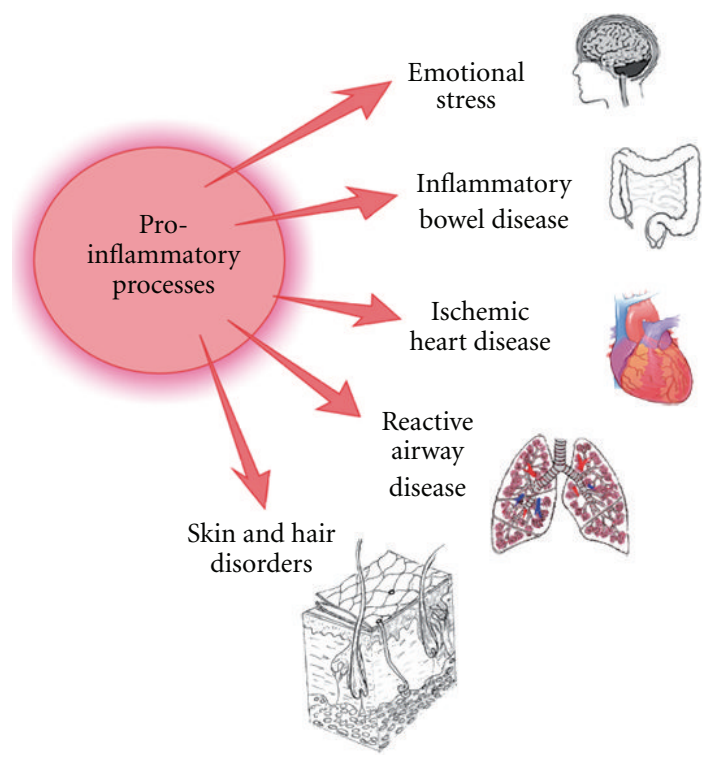

Figure 2: Many organ systems are affected by pro-inflammatory processes.

CCL17 is also over-expressed in stromal, endothelial and epithelial tissues in autoimmune disorders including the inflammatory bowel diseases, Crohn's disease and ulcerative colitis (UC) [28]. Finally, $\operatorname{LTB}(4)$ is a lipid inflammatory mediator derived from membrane phospholipids by the sequential actions of cytosolic phospholipase A2 (PLA2), 5lipoxygenase (5-LO) and leukotriene A(4) [LTA(4)] hydrolase. $\operatorname{LTB}(4)$, best known as a neutrophil chemoattractant, is now recognized to exert other important effects contributing to inflammatory and immune responses. Reinforcing the connection between androgen-mediated disorders and inflammatory processes, LTB(4) expression has been demonstrated in acne vulgaris [29].

While LPS caused upregulation of CXCL6 and LTB(4) at both doses as expected, we observed a modest downregulation of CCL17 at the higher dose of LPS. Likewise, in a recent study using the LPS challenge cell culture model, the authors noted that their responses might have also differed from predicted expectation based on the presence or absence of specific cell-surface receptors in $2 \mathrm{D}$ culture versus the 3D environment [30]. Thus, the observation that high-dose LPS itself partially lowers CCL17 expression does not change our interpretation, since the treatment represses expression beyond the level of high-dose LPS. The overall result of our experiment is that LPS challenge (at low dose) induces the expected increase in CCL17, and the treatment group demonstrates potent downregulation of CCL17.

A large body of evidence supports the involvement of pro-inflammatory processes in the development and progression of numerous disorders, including inflammatory bowel disease, ischemic heart disease and asthma (Figure 2) [18]. Chronic inflammation is recognized at the molecular and cellular levels as the final common pathway of many systemic and degenerative diseases, including those affecting the skin [31]. It has also been observed that inflammation, even that induced by emotional stress, plays a role in hair loss, and recent studies point to inflammation as a contributing factor to AGA [32]. In a murine model, the authors demonstrated that psychoemotional stress indeed alters actual HF cycling in vivo, that is, prematurely terminates the normal duration of active hair growth (anagen) [33]. Furthermore, inflammatory events deleterious to the HF are present in the HF environment of stressed mice (perifollicular macrophage cluster, excessive mast cell activation). It has also been shown experimentally that high-dose pro-inflammatory cytokines induce apoptosis of HF keratinocytes in vivo [34].

Histologically, sustained microscopic follicular inflammation with connective tissue remodeling is a noted cofactor in the complex etiology of AGA [35]. Disruption of HF homeostasis, via injury, insult or inflammation results in a shift from proliferation to apoptosis. Cumulatively, these findings underline the susceptibility HFs display when subjected to inflammatory insult.

In order to clarify the rationale leading to this study, L-carnitine, TA and LSESr operate through distinct, but potentially interrelated, biochemical and molecular mechanisms, with each representing an important component in the design of the test formula. Carnitine, a potent antiinflammatory agent, biosynthesized from the amino acids lysine and methionine is required for the transport of fatty acids from the cytosol into the mitochondria during the breakdown of lipids for the generation of metabolic energy [36]. Carnitine exists in two stereoisomers: its biologically active form is L-carnitine, while its enantiomer, D-carnitine, is biologically inactive [37].

Accumulating evidence suggests that L-carnitine may play a significant role in prevention and treatment of numerous diseases as well as protection from accelerated aging that result from oxygen free-radical damage, inflammation and glycation (non-enzymatic glycosylation). Documented benefits from L-carnitine include clinical improvement in patients diagnosed with diabetes, cardiovascular disease, hypertension, congestive heart failure, age-related deterioration of brain function and vision and immune function [38]. One line of research suggests that carnitine may possess the ability to promote hair growth in vitro by increasing energy supply to the rapidly prolife rating and the energyconsuming anagen hair matrix [39].

The naturally occurring antioxidant TA was first described as an essential cofactor for the conversion of pyruvate to acetyl-CoA, a critical step in respiration [40]. TA is now recognized as a compound with many biological functions, such as the modulation of pathogenic inflammatory events, including those in the skin [41].

In addition to the two constituents described above, the test compound also contains the LSESr derived from the fruit of saw palmetto. LSESr is highly enriched with fatty acids and phytosterols and has been used historically to treat urinary tract symptoms, including BPH [42]. LSESr has been shown to block both isoforms of 5-alpha reductase (types I and II) with the added benefit that, in contrast to finasteride, LSESr does not interfere with PSA (prostate-specific antigen) levels [43]. As previously noted, we were the first to clinically demonstrate the utility of LSESr against AGA [8]. 
While the genetic architecture of AGA has not yet been determined, it is clearly a polygenic disorder in which multiple genes, hormonal pathways and environmental factors contribute to phenotype. To date, DHT modulation via 5-AR blockade remains the only druggable target shown to ameliorate the progression of AGA [44]. An important feature of our hypothesis hinges on the enhanced efficacy of combining anti-inflammatory agents with 5-AR inhibitors.

A paradigm for enhanced performance via concomitant therapy already exists in the treatment of skin disease. For example, studies show that the addition of a topical corticosteroid to imidazole therapy increases the bioavailability and prolongs the activity of the antimycotic, while rapidly reducing inflammatory symptoms [45].

In several studies, a synergy between $\mathrm{TA}$ and vitamin $\mathrm{E}$ has been described, and potent antioxidant effects can be obtained when both antioxidants are simultaneously used. Specifically, recent findings showed that the combination of TA plus vitamin E effectively reduces oxidative damage in brain and cardiac ischemia as well as in other pathological events associated with tissue inflammation and secondary to the formation of reactive oxygen species (ROS) [46]. In in vitro and animal studies, L-carnitine, particularly when used in combination with TA, has been shown to reverse agerelated changes in numerous tissues, systems and pathways [47].

Since the clinical success rate for treatment of AGA with androgen blockade is limited, there is enormous unmet medical need for patients who are refractory to current therapy. Recognizing that inflammation contributes to a wide range of diseases, including those affecting the skin and hair, it is our hypothesis that the blockade of inflammation represents a new and potentially viable therapeutic avenue. We suggest that our data may offer new insight into the potential for combining anti-inflammatory compounds with 5-AR inhibitors for the treatment of AGA.

\section{Acknowledgments}

The authors are grateful for the technical support and advice from SuperArray Biosciences, Inc. In particular, we thank Dr Timothy Steppe for valuable and stimulating discussions. They thank Tiffany Devine, Marcy Kuentzel and David Frank at the Center for Functional Genomics, State University of New York (SUNY), Albany, NY, for their participation in preparation of the experimental protocols.

\section{References}

[1] S. Délos, C. Iehlé, P. M. Martin, and J. Raynaud, "Inhibition of the activity of 'basic' 5 alpha-reductase (type 1) detected in DU 145 cells and expressed in insect cells," The Journal of Steroid Biochemistry and Molecular Biology, vol. 48, pp. 347-352, 1994.

[2] E. Stoner, "The clinical effects of a $5 \alpha$-reductase inhibitor, finasteride, on benign prostatic hyperplasia," Journal of Urology, vol. 147, no. 5, pp. 1298-1302, 1992.

[3] P. Ekman, "Pharmacological treatment of benign prostatic hyperplasia with Finasteride: a clinical review," Archivos Espanoles de Urologia, vol. 47, no. 9, pp. 883-888, 1994.
[4] E. Koch, "Extracts from fruits of saw palmetto (Sabal serrulata) and roots of stinging nettle (Urtica dioica): viable alternatives in the medical treatment of benign prostatic hyperplasia and associated lower urinary tracts symptoms," Planta Medica, vol. 67, pp. 489-500, 2001.

[5] G. L. Plosker and R. N. Brogden, "Serenoa repens (Permixon). A review of its pharmacology and therapeutic efficacy in benign prostatic hyperplasia," Drugs and Aging, vol. 9, no. 5, pp. 379-395, 1996.

[6] K. D. Kaufman and R. P. Dawber, "Finasteride, a Type 2 $5 \alpha$-reductase inhibitor, in the treatment of men with androgenetic alopecia," Expert Opinion on Investigational Drugs, vol. 8, no. 4, pp. 403-415, 1999.

[7] K. D. Kaufman, E. A. Olsen, D. Whiting et al., "Finasteride in the treatment of men with androgenetic alopecia. Finasteride Male Pattern Hair Loss Study Group," Journal of the American Academy of Dermatology, vol. 39, pp. 578-589, 1998.

[8] N. Prager, K. Bickett, N. French, and G. Marcovici, "A randomized, double-blind, placebo-controlled trial to determine the effectiveness of botanically derived inhibitors of 5- $\alpha$-reductase in the treatment of androgenetic alopecia," Journal of Alternative and Complementary Medicine, vol. 8, no. 2, pp. 143-152, 2002.

[9] R. M. Trüeb, "Association between smoking and hair loss: another opportunity for health education against smoking?" Dermatology, vol. 206, pp. 189-191, 2003.

[10] A. Descazeaud, M. A. Rubin, M. Hofer et al., "BPH gene expression profile associated to prostate gland volume," Diagnostic Molecular Pathology, vol. 17, no. 4, pp. 207-213, 2008.

[11] R. M. Trüeb, "Molecular mechanisms of androgenetic alopecia," Experimental Gerontology, vol. 37, no. 8-9, pp. 981-990, 2002.

[12] P. Hirsso, U. Rajala, L. Hiltunen, J. Jokelainen, S. KeinänenKiukaanniemi, and S. Näyhä, "Obesity and low-grade inflammation among young finnish men with early-onset alopecia," Dermatology, vol. 214, no. 2, pp. 125-129, 2007.

[13] K. A. Greco and K. T. McVary, "The role of combination medical therapy in benign prostatic hyperplasia," International Journal of Impotence Research, vol. 20, no. 3, pp. S33-S43, 2008.

[14] K. T. McVary, "A review of combination therapy in patients with benign prostatic hyperplasia," Clinical Therapeutics, vol. 29, no. 3, pp. 387-398, 2007.

[15] F. D. Khan, S. Roychowdhury, R. Nemes, P. M. Vyas, P. M. Woster, and C. K. Svensson, "Effect of pro-inflammatory cytokines on the toxicity of the arylhydroxylamine metabolites of sulphamethoxazole and dapsone in normal human keratinocytes," Toxicology, vol. 218, no. 2-3, pp. 90-99, 2006.

[16] S. Huh, J. Lee, E. Jung et al., "A cell-based system for screening hair growth-promoting agents," Archives of Dermatological Research, vol. 301, no. 5, pp. 381-385, 2009.

[17] R. Moeller, J. Lichter, and B. Blömeke, "Impact of paraphenylenediamine on cyclooxygenases expression and prostaglandin formation in human immortalized keratinocytes (HaCaT)," Toxicology, vol. 249, no. 2-3, pp. 167-175, 2008.

[18] H. G. Yoo, I.-Y. Chang, H. K. Pyo et al., "The additive effects of minoxidil and retinol on human hair growth in vitro," Biological and Pharmaceutical Bulletin, vol. 30, no. 1, pp. 21-26, 2007.

[19] S.-S. Rho, S.-J. Park, S.-L. Hwang et al., "The hair growth promoting effect of Asiasari radix extract and its molecular regulation," Journal of Dermatological Science, vol. 38, no. 2, pp. 89-97, 2005.

[20] P. Chavan, K. Joshi, and B. Patwardhan, "DNA microarrays in herbal drug research," Evidence-Based Complementary and Alternative Medicine, vol. 3, no. 4, pp. 447-457, 2006. 
[21] H. Kobayashi, M. Ishii, S. Takeuchi et al., "Efficacy and safety of a traditional herbal medicine, Hochu-ekki-to in the longterm management of Kikyo (Delicate Constitution) patients with atopic dermatitis: a 6-month, multicenter, double-blind, randomized, placebo-controlled study," Evidence-Based Complementary and Alternative Medicine, vol. 7, no. 3, pp. 367-373, 2010.

[22] J. W. Cho, C. W. Kim, and K. S. Lee, "Modification of gene expression by melatonin in UVB-irradiated HaCaT keratinocyte cell lines using a cDNA microarray," Oncology Reports, vol. 17, no. 3, pp. 573-577, 2007.

[23] K. M. Lee, J. G. Lee, E. Y. Seo et al., "Analysis of genes responding to ultraviolet $\mathrm{B}$ irradiation of $\mathrm{HaCaT}$ keratinocytes using a cDNA microarray," British Journal of Dermatology, vol. 152, no. 1, pp. 52-59, 2005.

[24] M. A. Dayem, C. Moreilhon, L. Turchi et al., "Early gene expression in wounded human keratinocytes revealed by DNA microarray analysis," Comparative and Functional Genomics, vol. 4, no. 1, pp. 47-55, 2003.

[25] C. Watkins, A. McKellar, K. Jensen et al., "Development and validation of an oligonucleotide microarray for immuno-inflammatory genes of ruminants," Veterinary Research Communications, vol. 32, no. 8, pp. 647-657, 2008.

[26] L. A. Begley, S. Kasina, J. MacDonald, and J. A. Macoska, "The inflammatory microenvironment of the aging prostate facilitates cellular proliferation and hypertrophy," Cytokine, vol. 43, no. 2, pp. 194-199, 2008.

[27] B. Homey, S. Meller, T. Savinko, H. Alenius, and A. Lauerma, "Modulation of chemokines by staphylococcal superantigen in atopic dermatitis," Chemical Immunology and Allergy, vol. 93, no. 1, pp. 181-194, 2007.

[28] N. N. Kristensen, D. Brudzewsky, M. Gad, and M. H. Claesson, "Chemokines involved in protection from colitis by CD4+ CD25+ regulatory T cells," Inflammatory Bowel Diseases, vol. 12, no. 7, pp. 612-618, 2006.

[29] G. Zoumpopoulou, E. Tsakalidou, J. Dewulf, B. Pot, and C. Grangette, "Differential crosstalk between epithelial cells, dendritic cells and bacteria in a co-culture model," International Journal of Food Microbiology, vol. 131, no. 1, pp. 40-51, 2009.

[30] T. Alestas, R. Ganceviciene, S. Fimmel, K. Müller-Decker, and C. C. Zouboulis, "Enzymes involved in the biosynthesis of leukotriene B4 and prostaglandin E2 are active in sebaceous glands," Journal of Molecular Medicine, vol. 84, pp. 75-87, 2006.

[31] P. Libby, "Role of inflammation in atherosclerosis associated with rheumatoid arthritis," American Journal of Medicine, vol. 121, no. 10, pp. S21-S31, 2008.

[32] C. R. Thornfeldt, "Chronic inflammation is etiology of extrinsic aging," Journal of Cosmetic Dermatology, vol. 7, no. 1, pp. 78-82, 2008.

[33] R. Rückert, G. Lindner, S. Bulfone-Paus, and R. Paus, "Highdose proinflammatory cytokines induce apoptosis of hair bulb keratinocytes in vivo," British Journal of Dermatology, vol. 143, pp. 1036-1039, 2000.

[34] P. C. Arck, B. Handjiski, E. M. Peters et al., "Stress inhibits hair growth in mice by induction of premature catagen development and deleterious perifollicular inflammatory events via neuropeptide substance P-dependent pathways," American Journal of Pathology, vol. 162, pp. 803-814, 2003.

[35] Y. F. Mahé, B. Buan, N. Billoni et al., "Pro-inflammatory cytokine cascade in human plucked hair," Skin Pharmacology, vol. 9, no. 6, pp. 366-375, 1996.
[36] M. Inazu and T. Matsumiya, "Physiological functions of carnitine and carnitine transporters in the central nervous system," Nihon Shinkei, Seishin, Yakurigaku Zasshi, vol. 28, pp. 113-120, 2008.

[37] A. A. Spasov and I. N. IIezhitsa, "Stereopharmacology of carnitine," Ross Fiziol Zh Im I M Sechenova, vol. 91, no. 12, pp. 1469-1480, 2005.

[38] M. Janson, "Orthomolecular medicine: the therapeutic use of dietary supplements for anti-aging," Clinical Interventions in Aging, vol. 1, no. 3, pp. 261-265, 2006.

[39] K. Foitzik, E. Hoting, T. Förster, P. Pertile, and R. Paus, "LCarnitine-L-tartrate promotes human hair growth in vitro," Experimental Dermatology, vol. 16, no. 11, pp. 936-945, 2007.

[40] G. R. Seaman, "Role of thioctic acid in the transfer of acyl groups," Proceedings of the Society for Experimental Biology and Medicine, vol. 82, pp. 184-189, 1953.

[41] S. Salinthone, V. Yadav, DN Bourdette, and D. W. Carr, "Lipoic acid: a novel therapeutic approach for multiple sclerosis and other chronic inflammatory diseases of the CNS," Endocrine, Metabolic \& Immune Disorders-Drug Targets, vol. 8, pp. 132-142, 2008.

[42] A. C. Buck, "Is there a scientific basis for the therapeutic effects of serenoa repens in benign prostatic hyperplasia? Mechanisms of action," The Journal of Urology, vol. 172, pp. 1792-1799, 2004.

[43] F. K. Habib, M. Ross, C. K. Ho, V. Lyons, and K. Chapman, "Serenoa repens (Permixon) inhibits the 5alpha-reductase activity of human prostate cancer cell lines without interfering with PSA expression," International Journal of Cancer, vol. 114, pp. 190-194, 2005.

[44] J. F. Libecco and W. F. Bergfeld, "Finasteride in the treatment of alopecia," Expert Opinion on Pharmacotherapy, vol. 5, no. 4, pp. 933-940, 2004.

[45] B. Havlickova and M. Friedrich, "The advantages of topical combination therapy in the treatment of inflammatory dermatomycoses," Mycoses, vol. 51, supplement 4, pp. 16-26, 2008.

[46] Ó. González-Pérez, N. A. Moy-López, and J. Guzmán-Muñiz, "Alpha-tocopherol and alpha-lipoic acid. An antioxidant synergy with potential for preventive medicine," Revista de Investigacion Clinica, vol. 60, no. 1, pp. 58-67, 2008.

[47] S. Kumaran, S. Savitha, M. Anusuya Devi, and C. Panneerselvam, "L-carnitine and DL- $\alpha$-lipoic acid reverse the agerelated deficit in glutathione redox state in skeletal muscle and heart tissues," Mechanisms of Ageing and Development, vol. 125, no. 7, pp. 507-512, 2004. 


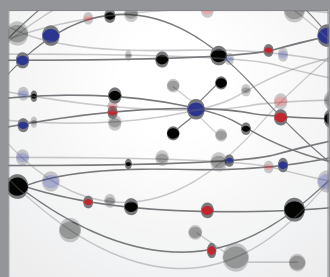

The Scientific World Journal
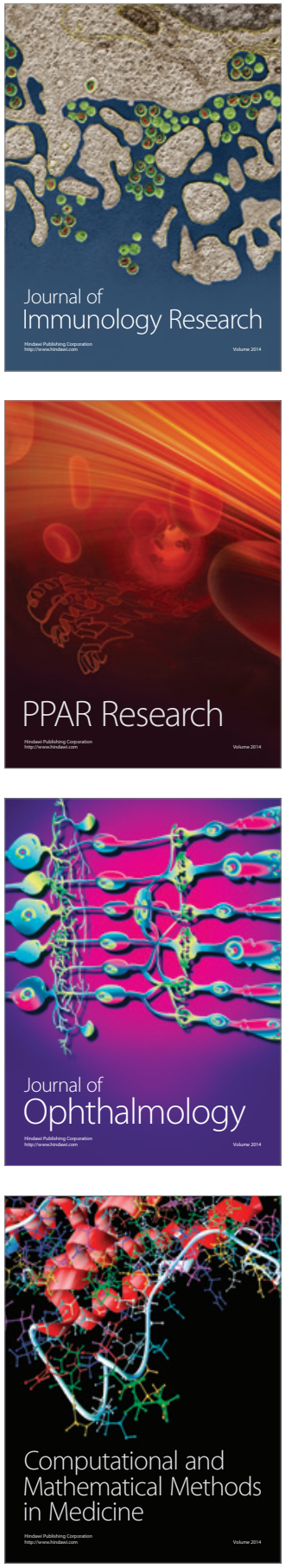

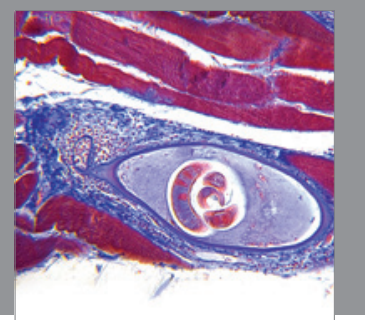

Gastroenterology

Research and Practice
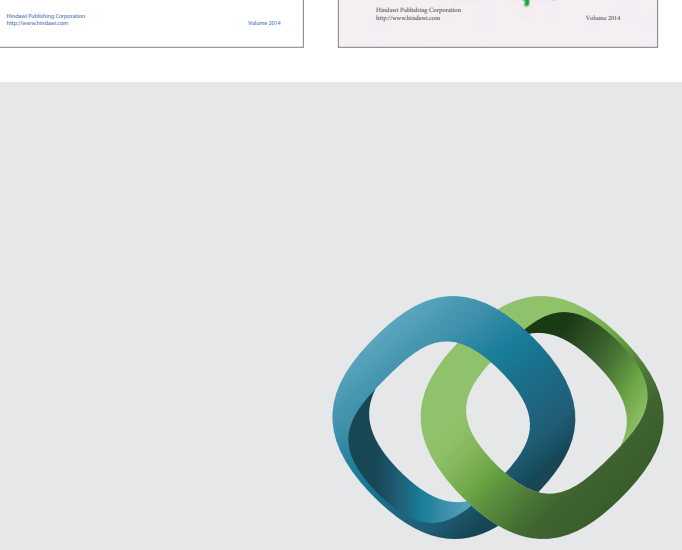

\section{Hindawi}

Submit your manuscripts at

http://www.hindawi.com
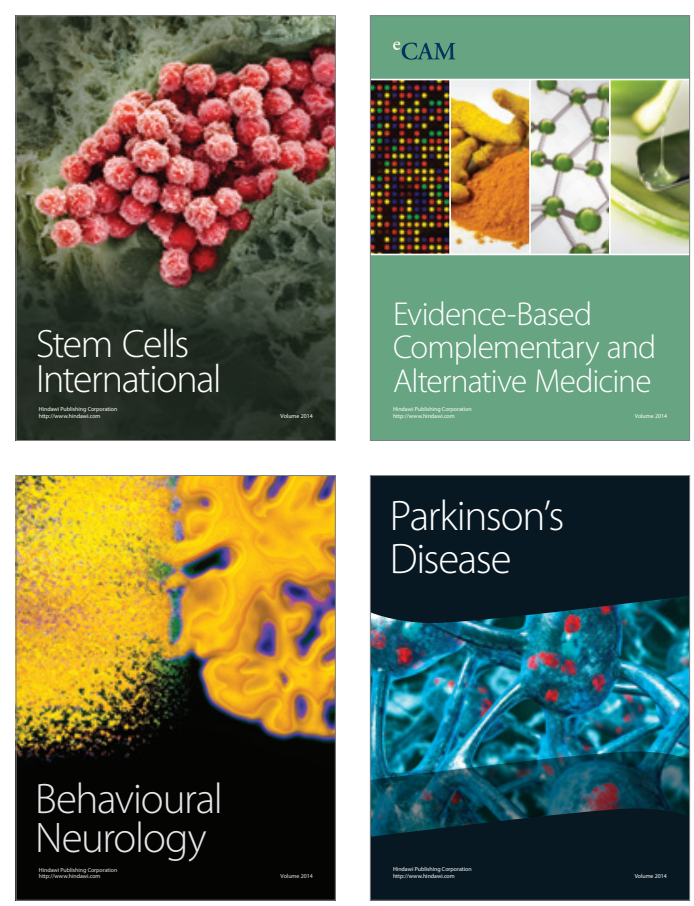

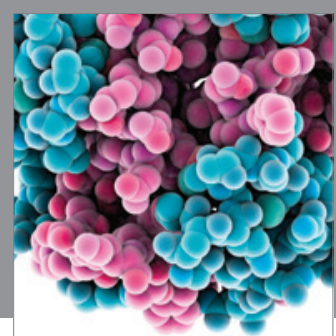

Journal of
Diabetes Research

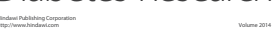

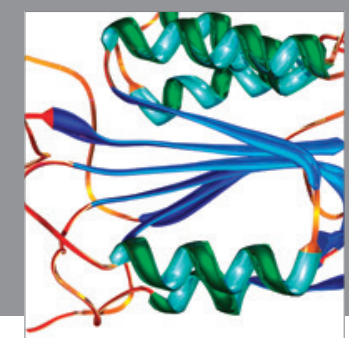

Disease Markers
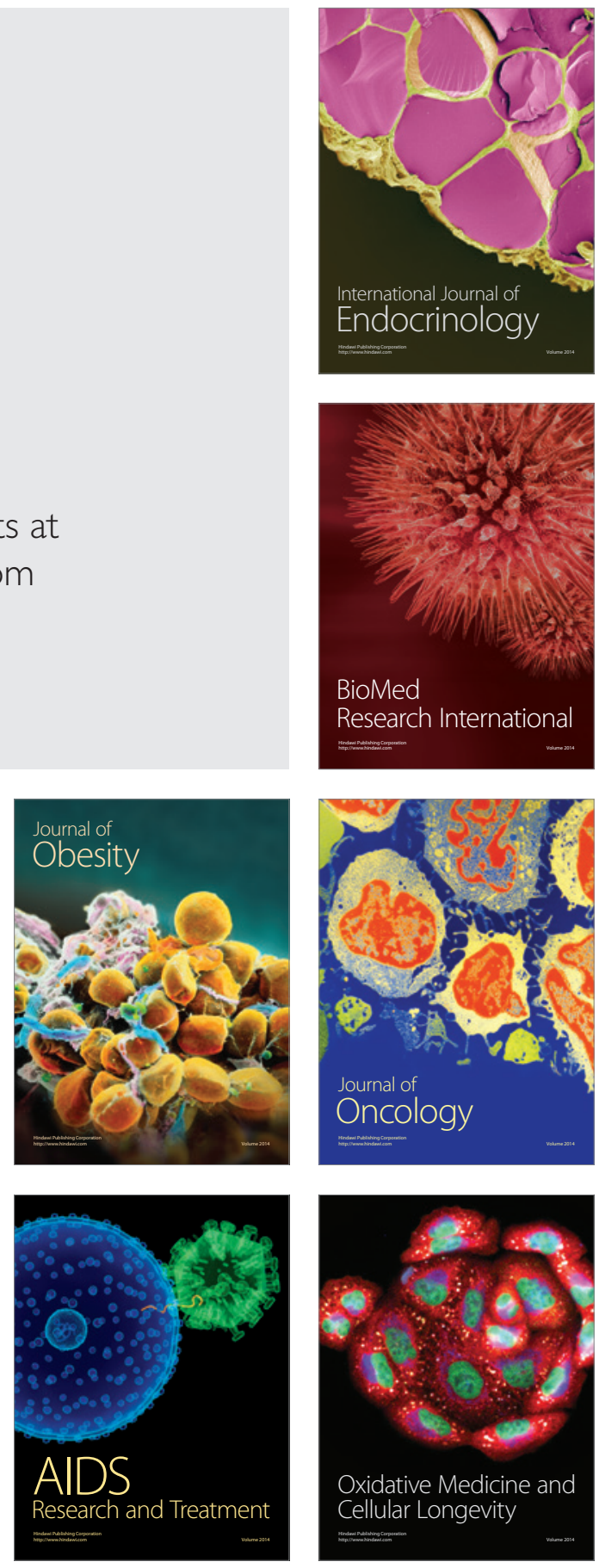